\title{
Dietary red palm oil reduces ischaemia-reperfusion injury in rats fed a hypercholesterolaemic diet
}

\author{
Maritza J. Kruger ${ }^{1}$, Anna-Mart Engelbrecht ${ }^{1 *}$, Johan Esterhuyse ${ }^{2}$, Eugene F. du Toit ${ }^{3}$ \\ and Jacques van Rooyen ${ }^{1}$ \\ ${ }^{1}$ Department of Physiological Sciences, University of Stellenbosch, Stellenbosch, 7600, South Africa \\ ${ }^{2}$ Faculty of Applied Sciences, Cape Peninsula University of Technology, Cape Town, South Africa \\ ${ }^{3}$ Department of Biomedical Sciences, University of Stellenbosch, Stellenbosch, South Africa
}

(Received 12 June 2006 - Revised 6 November 2006 - Accepted 6 November 2006)

\begin{abstract}
We have previously shown that dietary red palm oil (RPO) supplementation improves functional recovery in hearts subjected to ischaemia-reperfusion. However, little knowledge exists concerning the effects of RPO supplementation of a high-cholesterol diet on ischaemia-reperfusion injury. The signalling mechanisms responsible for RPO's effects in the presence of cholesterol also remain to be elucidated. Therefore, the aim of the present study was to examine the effects of RPO, given with a high-cholesterol diet, on mitogen-activated protein kinase (MAPK) phosphorylation and apoptosis. Long-Evans rats were fed a control diet, a control diet containing $2 \%$ cholesterol, or a control diet containing $2 \%$ cholesterol and $7 \mathrm{~g}$ RPO per kg (CRPO) for 5 weeks. Hearts were excised and mounted on an isolated working heart perfusion apparatus. Cardiac function was measured after which hearts were freeze-clamped and used to assess MAPK phosphorylation and to evaluate apoptosis. Cholesterol supplementation caused a poor aortic output (AO) recovery compared with the control group (35.5 (SEM 6.2) v. 55.4 (SEM 2.5) \%), but when RPO was added, the percentage AO increased significantly. The cholesterol group's poor AO was associated with a significant increase in p38-MAPK phosphorylation, whereas the CRPO-supplemented group showed as significant reduction in p38-MAPK phosphorylation when compared with the cholesterol-supplemented group. This significant reduction in p38-MAPK was also associated with reduced apoptosis as indicated by significant reductions in caspase- 3 and poly(ADP-ribose) polymerase cleavage.
\end{abstract}

Red palm oil: Mitogen-activated-protein kinase: Ischaemia: Reperfusion

CVD has become the leading cause of mortality and morbidity in Western countries. It is one of the most significant dietrelated health problems and there is a great deal of interest in how dietary fat composition influences the development of CVD. We have previously shown that dietary red palm oil (RPO) supplementation protects against ischaemiareperfusion-induced injury in the isolated perfused rat heart; however, the effects of RPO supplementation on ischaemiareperfusion-induced injury have not been investigated when supplemented with a high-cholesterol diet.

RPO is a natural oil obtained from oil palm fruit (Elaeis guineensis). It is high in palmitic (44\%) and oleic acid $(40 \%)$ with natural fat-soluble tocopherol, tocotrienol and carotonoids, which may act as antioxidants. Despite the high saturated fat content of RPO, several studies have demonstrated that RPO supplementation has beneficial or neutral effects on serum total cholesterol (Zhang et al. 1997a,b; Niyongabo et al. 1999; Nor Aini \& Suria, 2000). Other than its effect on lipoproteins, a few recent studies have shown that RPO has cellular signalling effects associated with protection and better recovery under conditions of ischaemia-reperfusion (Esterhuyse et al. 2005; Engelbrecht et al. 2006). However, the signalling events in hearts supplemented with RPO in the presence of cholesterol remain to be elucidated.

Mitogen-activated-protein kinase (MAPK) pathways are among the best characterised signalling pathways in the heart. The major regulatory enzymes of the MAPK pathways, namely extracellular signal-regulated protein kinases (ERK1/ 2), c-Jun $\mathrm{NH}_{2}$-terminal kinases (JNK) and $\mathrm{p} 38$, have been implicated in cell proliferation, differentiation and apoptosis (Bhattacharya et al. 2004). Furthermore, various studies have shown that MAPK are regulated in direct response to ischaemia-reperfusion-induced injury (Maulik et al. 1996; Bernabe et al. 2001; Bhattacharya et al. 2004).

It is important to note, however, that discrepancies exist regarding the functions of the different MAPK in the heart. JNK mainly acts in association with p38-MAPK, which also plays a critical role in apoptosis when stimulated by environmental stressors (Matsukawa et al. 2004). Unlike JNK though, p38-MAPK is highly expressed during ischaemia and reperfusion, whereas JNK is only activated during reperfusion

\footnotetext{
Abbreviations: ERK, extracellular signal-regulated protein kinase; JNK, c-Jun $\mathrm{NH}_{2}$-terminal kinase; MAPK, mitogen-activated-protein kinase; PARP, poly(ADPribose) polymerase; RPO, red palm oil; Tris, tri(hydroxymethyl)-aminomethane.

* Corresponding author: Dr A.-M. Engelbrecht, fax +27 218083145 , email ame@sun.ac.za
} 
(Whitmarsh et al. 1997). The dynamic balance between the growth factor-activated ERK and stress-activated JNK and p38-MAPK are important in determining whether a cell survives or undergoes apoptosis.

The protective effect of RPO against ischaemia-reperfusion-induced injury in the isolated perfused rat heart was observed in animals consuming standard rat chow which was low in cholesterol (Esterhuyse et al. 2005; Engelbrecht et al. 2006). In the present study we investigated whether RPO would counteract the negative effects of cholesterol in rats fed standard rat chow supplemented with $2 \%$ cholesterol. A previous study (Engelbrecht et al. 2006) also showed that RPO-induced protection of the heart against reperfusion injury was associated with changes in MAPK phosphorylation. Therefore, in the present study, we correlated the effect of RPO supplementation of a high-cholesterol diet with MAPK phosphorylation and apoptosis.

\section{Materials and methods}

\section{Antibodies and chemicals}

All primary phospho-specific antibodies were purchased from Cell Signaling Technology (New England BioLabs, Ipswich, MA, USA), secondary antibodies from Amersham Biosciences (Piscataway, NJ, USA), and preGOLD prestained protein marker IV from PEQLAB Biotechnology GmbH (Erlangen, Germany). Chemiluminescence detection kits were purchased from Amersham Biosciences, whereas Western blotting systems used were obtained from Bio-Rad Laboratories Inc. (Hercules, CA, USA) and all chemicals from commercial companies.

\section{Experimental model}

All animals used received humane care in accordance with the Principle of Laboratory Animal Care of the National Society of Medical Research and the National Institutes of Health Guide for the Care and Use of Laboratory Animals of the National Academy of Sciences (National Institutes of Health publication no. 80-23, revised 1978). Long-Evans rats aged 7 weeks were randomly allocated to three groups according to the dietary supplementation they received: group 1, standard rat chow (control group); group 2, standard rat chow enriched with $2 \%$ cholesterol; group 3, standard rat chow enriched with $2 \%$ cholesterol and RPO (7 g/kg diet) (Table 1).
The rats were fed a standard rat chow diet or $2 \%$ cholesterol-enriched diet (based on previous studies by Giricz et al. 2003) for 6 weeks. The approximate energy and macronutrient content of the two diets are indicated in Table 1 . The rat chow was supplied by Atlas Animal Foods (Cape Town, South Africa) and regularly analysed to monitor possible variations between batches. Rats of the control group consumed an average of $25 \mathrm{~g}$ standard rat chow/d, containing $0.625 \mathrm{~g}$ fat, which provided $8.7 \%$ of the energy intake. Protein intake was $4.5 \mathrm{~g}$ ( $28 \%$ of energy intake). In the additional group the cholesterol-enriched rat chow diet was supplemented with RPObaking fat ( $7 \mathrm{~g}$ RPO per $\mathrm{kg}$ diet) every morning for 6 weeks. Thus, there was a $21 \%$ increase in fat intake in the RPO-supplemented experimental group. The RPO used in the present study provided an additional $70.0 \mu \mathrm{g}$ carotenoids and $87.5 \mu \mathrm{g}$ vitamin $\mathrm{E}$ (tocotrienols and tocopherols) to that present in the standard rat chow diet (antioxidant nutrient status of standard rat chow diet not provided by supplier due to confidentiality) (Nagendran et al. 2000). There were no significant differences in the serum total cholesterol or the HDL-cholesterol after the 6-week cholesterol and RPO supplementation period. Serum concentrations of TAG were increased in the RPO- and cholesterol-supplemented groups after 6 weeks on the diet (0.056 (SEM 0.07) and 0.53 (SEM 0.08) $\mathrm{mmol} / \mathrm{l}$, respectively before the 6-week diet and 0.87 (SEM 0.08) and 1.05 (SEM 0.19 ) $\mathrm{mmol} / \mathrm{l} ; P<0.05$, respectively after the 6week diet). In the control group it was 0.54 (SEM 0.07) $\mathrm{mmol} / \mathrm{l}$ before and 0.83 (SEM 0.09) $\mathrm{mmol} / \mathrm{l}$ after the 6-week diet (Esterhuyse et al. 2005).

Rats weighing 300-400 g were anaesthetised with sodium thiopentone before the hearts were rapidly excised and placed in ice-cold Krebs-Henseleit buffer. Hearts were transferred to the standard working heart perfusion apparatus and perfused with a Krebs-Henseleit buffer ( $\mathrm{pH}$ 7.4) containing: $119 \mathrm{~mm}-\mathrm{NaCl}, \quad 24.9 \mathrm{~mm}-\mathrm{NaHCO}_{3}, \quad 4.74 \mathrm{~mm}-\mathrm{KCl}, \quad 1.19 \mathrm{~mm}-$ $\mathrm{KH}_{2} \mathrm{PO}_{4}, 0.6 \mathrm{~mm}-\mathrm{MgSO}_{4}, 0.59 \mathrm{~mm}-\mathrm{Na}_{2} \mathrm{SO}_{4}, 1.25 \mathrm{~mm}-\mathrm{CaCl}_{2}$ and $10 \mathrm{~mm}$-glucose. The buffer was oxygenated and kept at pH 7.4 by gassing with $\mathrm{O}_{2}-\mathrm{CO}_{2}(95: 5, \mathrm{v} / \mathrm{v})$. The aorta was cannulated and retrograde perfusion was initiated. During this initial perfusion in the Langendorff mode, the opening to the left atrium was cannulated. Following a 5 min stabilisation period in the Langendorff mode, hearts were switched to the working heart mode for $20 \mathrm{~min}$. The temperature of both the perfusate and the air surrounding the heart was thermostatically controlled and checked at regular intervals

Table 1. Approximate energy and macronutrient contents of rat diets

\begin{tabular}{lcc}
\hline Nutrient & Standard rat chow diet (control) & $\begin{array}{c}\text { Standard rat chow diet supplemented } \\
\text { with red palm oil }\end{array}$ \\
\hline Energy $(\mathrm{kJ})$ & 272.5 & $277 \cdot 6$ \\
Total non-structural carbohydrates $(\mathrm{g})$ & 8.375 & 8.375 \\
Total protein $(\mathrm{g})$ & 4.5 & 4.5 \\
Total fat $(\mathrm{g})$ & 0.625 & 0.758 \\
Total SFA $(\mathrm{g})$ & 0.139 & 0.206 \\
Total MUFA $(\mathrm{g})$ & 0.168 & 0.219 \\
Total PUFA (g) & 0.297 & 0.312 \\
Total $n$-6 PUFA $(\mathrm{g})$ & 0.247 & 0.248 \\
Total $n$-3 PUFA $(\mathrm{g})$ & 0.049 & 0.061 \\
\hline
\end{tabular}

* $25 \mathrm{~g}$ standard rat chow per $\mathrm{d}$.

$\dagger 25 \mathrm{~g}$ standard rat chow plus $0.175 \mathrm{~g}$ red palm oil per $\mathrm{d}$. 
to ensure that the temperature was maintained at $37^{\circ} \mathrm{C}$ irrespective of coronary flow. Hearts were then subjected to $25 \mathrm{~min}$ of total global ischaemia. At the end of ischaemia, hearts were reperfused in the Langendorff mode for $10 \mathrm{~min}$, followed by $15 \mathrm{~min}$ working heart perfusion. In order to reduce the incidence of reperfusion arrhythmias, $2 \%$ lignocaine solution was used for the initial $3 \mathrm{~min}$ of reperfusion of all hearts. To assess myocardial MAPK activity, hearts were freeze-clamped with Wollenberger clamps pre-cooled in liquid $\mathrm{N}_{2}$ at the end of the pre-ischaemic working heart perfusion (four rats per group), after $10 \mathrm{~min}$ ischaemia (four rats per group) and $10 \mathrm{~min}$ into reperfusion (four rats per group), and samples were stored at $-80^{\circ} \mathrm{C}$ (Fig. 1).

\section{Functional parameters measured}

Coronary and aortic flow rates were measured by collecting $1 \mathrm{~min}$ samples of the respective effluents $25 \mathrm{~min}$ into the pre-ischaemic perfusion and $25 \mathrm{~min}$ into reperfusion. Aortic output recovery was calculated by dividing the aortic output measured after ischaemia by that measured before ischaemia and expressing these values as percentage recovery (Fig. 1).

\section{Western blot analysis}

Cardiac MAPK and protein kinase B/Akt as well as caspase-3 and poly(ADP-ribose) polymerase (PARP) protein were extracted with a lysis buffer containing: $20 \mathrm{mM}$-tri(hydroxymethyl)-aminomethane (Tris), $20 \mathrm{~mm}$ - $p$-nitrophenylphosphate, $1 \mathrm{~mm}$-EGTA, $50 \mathrm{~mm}$-sodium fluoride, $0 \cdot 1 \mathrm{~mm}$-sodium orthovanadate, $1 \mathrm{~mm}$-phenylmethyl sulfonyl fluoride, $1 \mathrm{~mm}$-dithiothreitol, aprotinin $(10 \mu \mathrm{g} / \mathrm{ml})$ and leupeptin $(10 \mu \mathrm{g} / \mathrm{ml})$. The tissue lysates were diluted in Laemmli sample buffer, boiled for $5 \mathrm{~min}$ and $10 \mu \mathrm{g}$ (MAPK and protein kinase B/Akt) or $50 \mu \mathrm{g}$ protein (caspase-3 and PARP) were separated by $10 \%$ PAGESDS-gel electrophoresis. The lysate protein content was determined using the Bradford technique (Bradford, 1976). The separated proteins were transferred to a PVDF membrane (Immobilon $^{\mathrm{TM}} \mathrm{P}$; Millipore Corp., Bedford, MA, USA). These membranes were routinely stained with Ponceau Red for visualisation of proteins. Non-specific binding sites on the membranes were blocked with $5 \%$ fat-free milk in Tris-buffered saline$0.1 \%$ Tween 20 and then incubated with the primary antibodies that recognise phosphospecific ERK p42/p44 $\left(\mathrm{Thr}^{202} / \mathrm{Tyr}^{204}\right)$, p38-MAPK $\left(\mathrm{Thr}^{180} / \mathrm{Tyr}^{182}\right)$, JNK p54/p46 $\left(\mathrm{Thr}^{183} / \mathrm{Tyr}^{185}\right)$, protein kinase B (Ser ${ }^{473}$ and $\mathrm{Thr}^{308}$ ), caspase-3 (p17 fragment pAb) and PARP ( $\mathrm{p} 85$ fragment $\mathrm{pAb}$ ). Membranes were subsequently washed with large volumes of Tris-buffered saline-0.1\% Tween $20(5 \times 5 \mathrm{~min})$ and the immobilised antibody conjugated with a diluted horseradish peroxidase-labelled secondary antibody (Amersham Life Science). After thorough washing with Tris-buffered saline- $0.1 \%$ Tween 20 , membranes were covered with $\mathrm{ECL}^{\mathrm{TM}}$ detection reagents and quickly exposed to an autoradiography film (Hyperfilm ECL, RPN 2103; Amersham Biosciences) to detect light emission through a non-radioactive method (ECL ${ }^{\mathrm{TM}}$ Western blotting). Films were densitometrically analysed (UN-SCAN-IT; Silk Scientific Corporation (SilkScience), USA, version 5.1) and phosphorylated protein values were corrected for minor differences in protein loading, if required. Experiments were performed (data not shown) to ensure that all signals were within the linear range of detection on the autoradiographs under our assay and gel-loading conditions.

\section{Data analysis}

Data are presented as mean values with their standard errors. Statistical significance was determined in multiple comparisons among independent groups of data in which ANOVA and Bonferroni's post hoc test indicated significant differences. $P<0.05$ was considered statistically significant.

\section{Results}

Aortic output recovery

We used aortic output recovery as an indirect index of the severity of ischaemia-reperfusion injury. Cholesterol supplementation decreased aortic output recovery when compared with the control group (35.5 (SEM 6.2) v. 55.4 (SEM 2.5) \%). However, when RPO was added to the cholesterol diet, the percentage aortic output recovery was significantly increased when compared with the cholesterol group (63.2 (SEM 3.1) \%; P<0.05) (Fig. 2).

Effect of red palm oil supplementation on the phosphorylation of p38, c-Jun $\mathrm{NH}_{2}$-terminal kinase and extracellular

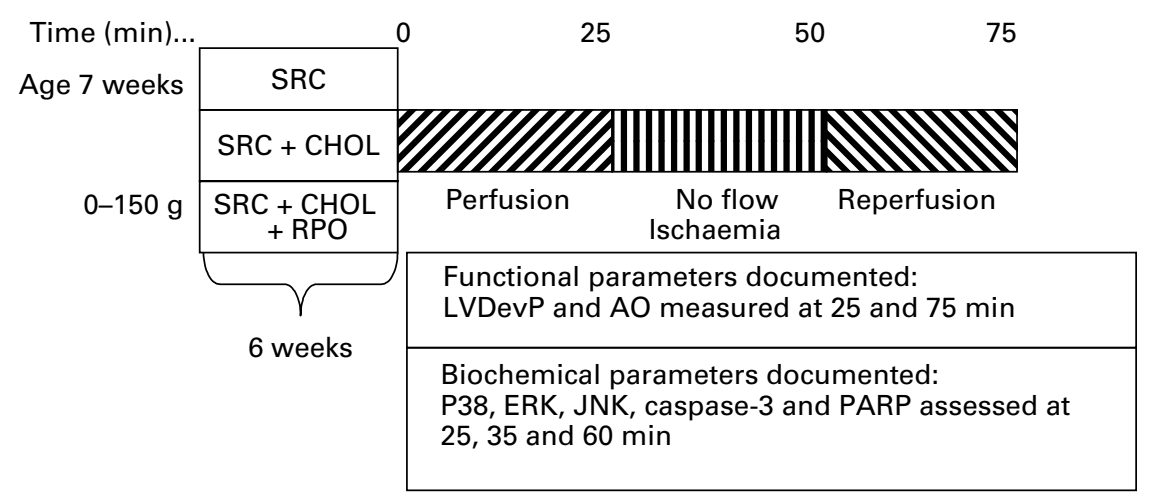

Fig. 1. A diagrammatic representation of the rat feeding programme and heart perfusion protocol employed for the study. SRC, standard rat chow; $\mathrm{SRC}+\mathrm{CHOL}$, standard rat chow enriched with $2 \%$ cholesterol; SRC + CHOL + RPO, standard rat chow enriched with $2 \%$ cholesterol and red palm oil; LVDevP, left ventricular developed pressure; $\mathrm{AO}$, aortic output; ERK, extracellular signal-regulated protein kinase; JNK, c-Jun $\mathrm{NH}_{2}$-terminal kinase; PARP, poly(ADP-ribose) polymerase. 


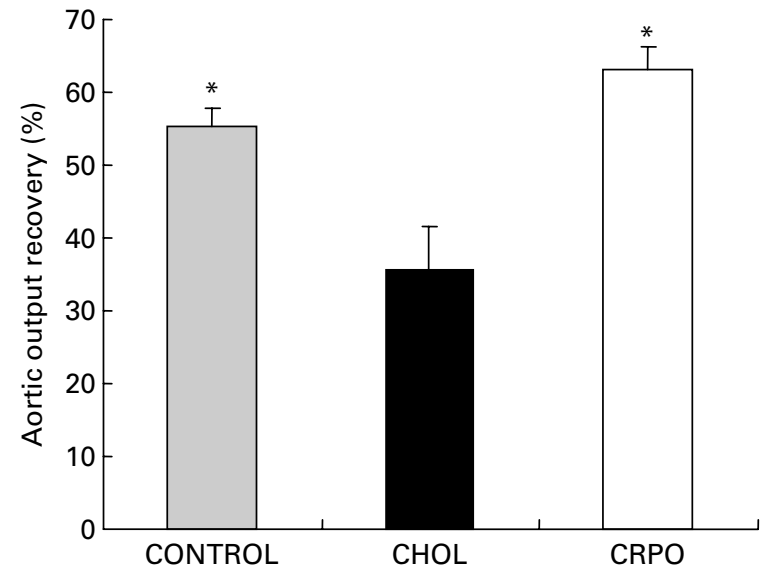

Fig. 2. The percentage aortic output recovery of cholesterol (CHOL)-supplemented hearts $v$. cholesterol-red palm oil (CRPO)-supplemented hearts $v$. control hearts. Data are means for seven independent experiments, with their standard errors represented by vertical bars. * Mean value was significantly different from that of the $\mathrm{CHOL}$ group $(P<0.05)$.

signal-regulated protein kinase in cholesterol-fed rat hearts subjected to ischaemia and reperfusion

Phosphorylation of p38, JNK (p46/p54-MAPK) and ERK1/ 2 (p42/p44-MAPK) was determined by Western blotting using phospho-specific antibodies. There was a significant increase in p38 phosphorylation in the control group during reperfusion $v$. baseline perfusion before ischaemia (1.67 (SEM 0.05)-fold; $P<0 \cdot 01$ ). As shown in Fig. 3 (A), p38 phosphorylation was significantly increased in the control group $v$. the cholesterol group during both ischaemia and reperfusion (0.86 (SEM 0.03)- to 3.05 (SEM 0.19)-fold; $P<0.001$ for ischaemia and 1.68 (SEM 0.05)- to 4.05 (SEM 0.29)-fold; $P<0.001$ for reperfusion). Ischaemia-reperfusion also caused significant increases in p38 phosphorylation in the cholesterol-RPO group when compared with the cholesterol group (0.88 (SEM 0.20 )- to 3.05 (SEM 0.19)-fold; $P<0.001$ for ischaemia and 2.07 (SEM 0.26)- to 4.05 (SEM 0.29)-fold; $P<0.001$ for reperfusion). There was a significant increase in ERK44/42 phosphorylation in the control group during reperfusion $v$. the baseline perfusion group (4.02 (SEM 0.51)-fold; $P<0.001$ for ERK44 and 3.11 (SEM 0.67)-fold; $P<0.001$ for ERK 42). ERK44 and ERK 42 phosphorylation was significantly increased in the cholesterol-RPO group during reperfusion when compared with the cholesterol group (1.42 (SEM 0.20)to 2.60 (SEM 0.76)-fold; $P<0.05$ for ERK44 and 1.55 (SEM 0.19)- to 3.33 (SEM 0.03)-fold; $P<0.001$ for ERK42). When compared with the control group, the phosphorylation of both subunits in the cholesterol group was significantly lower (1.42 (SEM 0.20)- to 4.02 (SEM 0.52)-fold; $P<0.001$ for ERK44 and 1.55 (SEM 0.19)- to 3.12 (SEM 0.67)-fold; $P<0.001$ for ERK42). No statistically significant differences occured between the cholesterol-RPO and control groups in the ERK42 fraction, but with regard to the ERK44 fraction, a significant reduction in ERK phosphorylation was found (2.60 (SEM 0.76)- to 4.02 (SEM 0.52)-fold; $P<0.01$ ). There was a significant increase in JNK54/46 phosphorylation in the control group during reperfusion $v$. the baseline perfusion group (1.39 (SEM 0.04)-fold; $P<0.001$ for JNK54 and 1.29 (SEM 0.09)-fold; $P<0.01$ for JNK 46). Dietary RPO supplementation in the presence of cholesterol caused a significant decrease in phosphorylation of both JNK54 and JNK46 during reperfusion when compared with the cholesterol group (1.45 (SEM 0.09)- to 1.21 (SEM 0.04)-fold; $P<0.001$ for JNK54 and 1.61 (SEM 0.08)- to 1.23 (SEM 0.04)-fold; $P<0.001$ for JNK46). Cholesterol supplementation, on the other hand, only caused a significant increase in the JNK46 fraction when compared with the control group (1.61 (SEM 0.08) to 1.30 (SEM 0.10); $P<0.001$ ), while the cholesterol-RPO group differed significantly from the control group in the JNK54 fraction during reperfusion (1.21 (SEM 0.04)- to 1.4 (SEM 0.04)-fold; $P<0 \cdot 01$ ). (Figs. 3 (A), (B) and (C))

The effect of red palm oil supplementation on caspase- 3 activation and poly(ADP-ribose) polymerase cleavage in cholesterol-fed rat hearts subjected to ischaemia and reperfusion

In Fig. 4 (A), total caspase-3 was measured during perfusion, ischaemia and reperfusion of the control, cholesterol and cholesterol-RPO groups. All groups showed a marked reduction in total caspase- 3 during ischaemia and reperfusion compared with baseline perfusion. An increase in total caspase- 3 is associated with less cleaved caspase- 3 and thus a reduction in apoptosis. The expression of total caspase- 3 in the cholesterol-RPO group was significantly higher compared with the control and cholesterol group during both ischaemia and reperfusion (0.71 (SEM 0.02)- to 0.28 (SEM 0.06)-fold; $P<0.001$ for control ischaemia and 0.24 (SEM 0.01)-fold; $P<0.001$ for cholesterol ischaemia; 0.56 (SEM 0.06)- to 0.27 (SEM 0.01)-fold; $P<0.001$ for control reperfusion and 0.42 (SEM 0.05)-fold; $P<0.01$ for cholesterol reperfusion). It is only during reperfusion that a significant difference in caspase cleavage was observed between the control and cholesterol group $(P<0 \cdot 01)$. In Fig. 4 (B), cleaved PARP was measured during perfusion, ischaemia and reperfusion of the control, cholesterol and cholesterol-RPO groups. A significant increase in cleaved PARP was found in both the cholesterol-RPO and cholesterol groups during ischaemia when compared with the control group $(P<0.001$ for both), where the amount of cleaved PARP in the cholesterol-RPO supplemented group was significantly lower than the cholesterol group (1.74 (SEM 0.04)- to 1.40 (SEM 0.11)-fold; $P<0.001$ ). During reperfusion an even greater amount of cleaved PARP was observed in all the groups, where a significant difference was found between all three groups $(P<0 \cdot 001)$.

\section{Discussion}

We have demonstrated for the first time that RPO supplementation of a cholesterol-enriched diet offers protection against ischaemia-reperfusion-induced injury in the isolated perfused working heart as reflected by improved myocardial functional recovery when compared with the cholesterol-supplemented control group (Fig. 2). This decreased myocardial functional recovery in the cholesterol-supplemented groups was also observed by Golino et al. (1987), who demonstrated that hypercholesterolaemia caused an increase in myocardial infarct size in rabbits after $30 \mathrm{~min}$ of ischaemia. Furthermore, Ferdinandy et al. (1997) demonstrated for the first time that the cardioprotection conferred by classical preconditioning was lost when rats became hypercholestrolaemic. Kyriakides et al. 

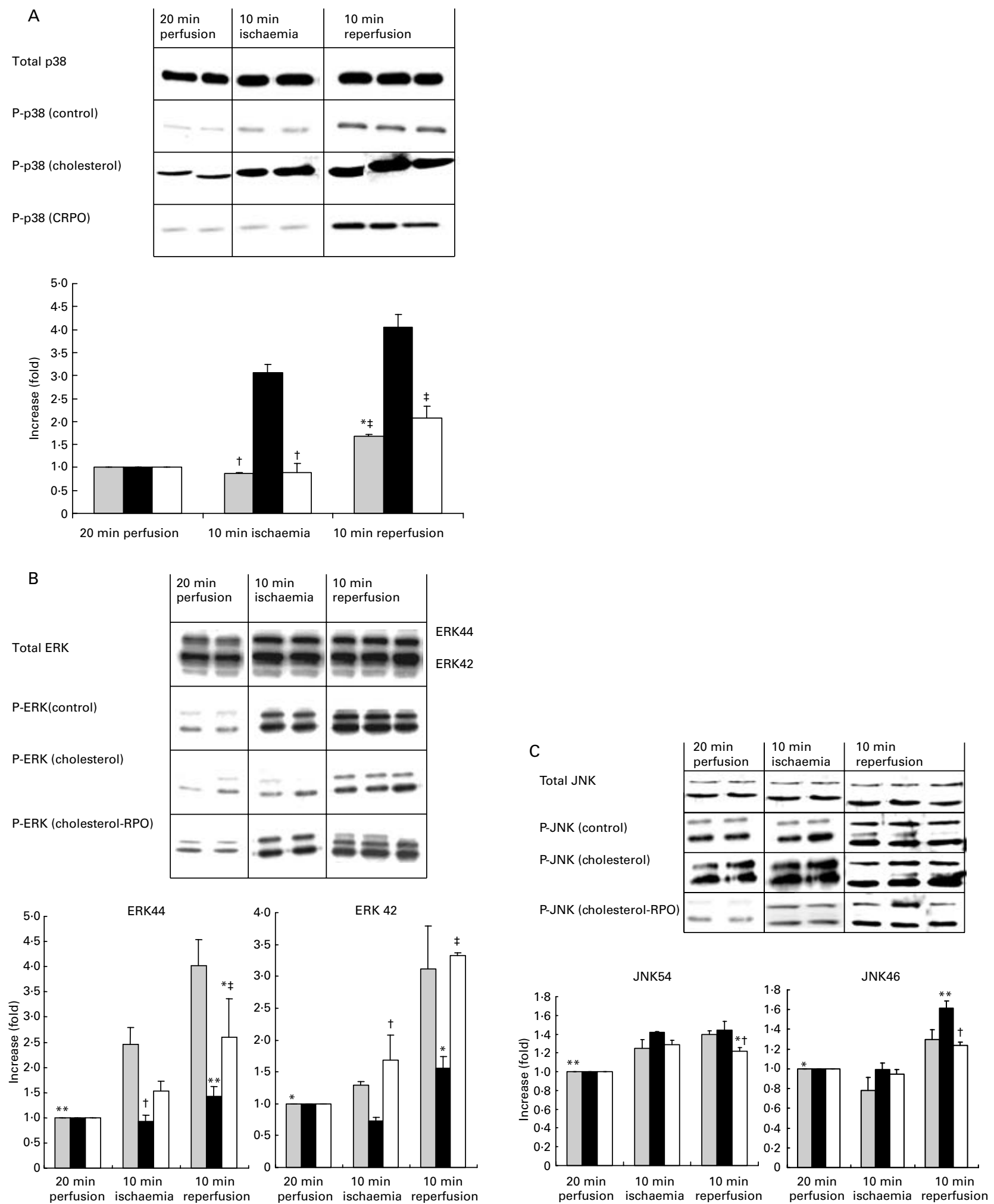

Fig. 3. (A) The effect of red palm oil (RPO) supplementation on the phosphorylation of p38-mitogen-activated-protein kinase (MAPK) in cholesterol-fed rat hearts subjected to ischaemia and reperfusion. Samples were analysed by Western blotting with phospho-specific antibodies recognising dual phosphorylated MAPK.

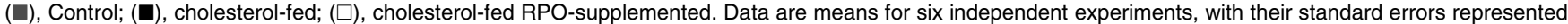
by vertical bars. * Mean value was significantly different from that of the 20 min perfusion control $(P<0.01)$. $\dagger$ Mean value was significantly different from that of the $10 \mathrm{~min}$ ischaemia cholesterol condition $(P<0.001)$. ¥ Mean value was significantly different from that of the 10 min reperfusion cholesterol condition $(P<0.001)$. (B) The effect of RPO supplementation on the phosphorylation of extracellular signal-regulated protein kinase (ERK) in cholesterol-fed rat hearts subjected to ischaemia and reperfusion. Samples were analysed by Western blotting with phospho-specific antibodies recognising dual phosphorylated MAPK. Data are means for six independent experiments, with their standard errors represented by vertical bars. ERK44: mean value was significantly different from that of the 10 min reperfusion control: ${ }^{\star} P<0.01,{ }^{\star \star} P<0.001$; † mean value was significantly different from that of the 10 min ischaemia control $(P<0.01)$; $\ddagger$ mean value was 
(2002) also showed that the anti-ischaemic effect of preconditioning is abolished in the presence of hypercholesterolaemia, emphasising the necessity of developing new cardioprotective drugs capable of preventing increased susceptibility of hearts to ischaemic stress, resulting in decreased functional recovery. Although there appears to be some conflicting data in the literature, the majority of studies show that hyperlipidaemia, independently from the development of coronary atherosclerosis, worsens the outcome of ischaemia-reperfusion injury and attenuates the cardioprotective effect of preconditioning (for a review, see Ferdinandy, 2003).

Engelbrecht et al. (2006) previously demonstrated that RPO supplementation offered significant protection against ischaemia-reperfusion-induced injury in the isolated perfused heart, but no evidence exists for RPO-induced protection in a cholesterol-supplemented diet. Although some evidence exist for an effect of some of the major components of RPO on cardiac function, it is still not clear whether the beneficial effect of RPO can be attributed to one single component or if it is the combined effect of the ingredients of RPO that induced the protection. For example, Serbinova et al. (1992) showed that RPO vitamin E was more efficient than tocopherols in protecting against ischaemia-reperfusion injury in the isolated perfused rat heart. Furthermore, Bilgin-Karabulut et al. (2001) showed that pre-treatment with a combination of vitamins $\mathrm{A}$ and $\mathrm{E}$ gave protection against venous ischaemia-reperfusion-induced injury. Interestingly, these vitamins were not effective when used as single agents. On the other hand, Meehan \& Higgins (1994) demonstrated that oleic acid (another major component of RPO) improved functional recovery in ischaemic-reperfused rat hearts.

Various signal transduction pathways are activated in the heart in response to ischaemia-reperfusion-induced injury. It has also been suggested that the MAPK are important regulators of apoptosis in response to myocardial ischaemia-reperfusion (Hreniuk et al. 2001). We characterised the phosphorylation pattern of the three major MAPK subfamilies after 20 min of perfusion, $10 \mathrm{~min}$ of ischaemia and $10 \mathrm{~min}$ of reperfusion and correlated it with markers of apoptosis at the same time points. Our data show that reperfusion in control hearts caused significant increases in p38, ERK and JNK phosphorylation compared with the 20 min baseline perfusion control groups. This is in agreement with results of Bogoyevitch et al. (1996) and Marais et al. (2001) who reported attenuation of MAPK phosphorylation during ischaemia-reperfusion. This increase in MAPK phosphorylation also correlated with an increase in apoptosis demonstrated by caspase-3 and PARP cleavage in our model.

We have demonstrated that dietary cholesterol supplementation significantly increased p38 phosphorylation during ischaemia and reperfusion compared with the control group
(Fig. 2 (A)) and that RPO supplementation caused a significant decrease in $\mathrm{p} 38$ phosphorylation. This down regulation of $\mathrm{p} 38$ with RPO supplementation was associated with improved function of the isolated perfused heart after ischaemia-reperfusion-induced injury. Despite reports to the contrary (Weinbrenner et al. 1997), most evidence (as well as our own data) supports the concept that p38 activation is harmful to the ischaemic heart (Bogoyevitch et al. 1996; Mackay \& Mochly-Rosen, 2000; Engelbrecht et al. 2004). These opposing results can be attributed to the different isoforms ( $\alpha$ and $\beta$ ) expressed in the heart (Saurin et al. 2000), which appear to mediate opposing functions. The p38 $\alpha$ isoform is implicated in apoptosis, whereas p38 $\beta$ is anti-apoptotic (Minet et al. 2001).

In our model, JNK phosphorylation was also significantly increased during reperfusion in the cholesterol-supplemented group but was attenuated by dietary RPO supplementation. This is in agreement with other results which showed that JNK phosphorylation appears to be pro-apoptotic in many cell types; however, its exact role in regulating cell death is still unclear (Wang et al. 1998; Obata et al. 2000; Park et al. 2000; Hreniuk et al. 2001).

Dietary RPO supplementation of the cholesterol-enriched diet also significantly increased ERK phosphorylation when compared with the cholesterol-supplemented group. It is suggested that the ERK pathway is required for survival signalling in response to ischaemia-reperfusion in cardiomyocytes (Abe et al. 2000). Among the substrates of ERK, p90 ribosomal S6 kinase (p90RSK) is a ubiquitous and versatile mediator of ERK signalling and one of its functions include the phosphorylation of the pro-apoptotic protein BAD at serine 112, which specifically suppresses BAD-mediated apoptosis (Bonni et al. 1999).

It was also demonstrated that the increase in functional recovery induced by RPO supplementation was associated with a significant inhibition of apoptosis in our model. Apoptosis has been consistently observed in the myocardium after ischaemia-reperfusion-induced injury and may represent a direct mechanism by which myocytes are killed (Yue et al. 2000; Hreniuk et al. 2001). Indeed, ischaemia-reperfusioninduced injury resulted in the cleavage of PARP, a phenomenon that is well known to result from caspase-3 activation. Inhibition of apoptosis by RPO may, therefore, offer a unique approach towards the amelioration of ischaemiareperfusion-induced injury.

The present results have shown that dietary RPO supplementation significantly protected the heart from the adverse effects of a high-cholesterol diet. Our data suggest that its beneficial effects are associated with decreased p38 and JNK phosphorylation and increased ERK phosphorylation which may contribute to the decrease in apoptosis in our model.

significantly different from that of the $10 \mathrm{~min}$ reperfusion cholesterol condition $(P<0.05)$. ERK42: * mean value was significantly different from that of the 10 min reperfusion control $(P<0.001)$; $†$ mean value was significantly different from that of the 10 min ischaemia cholesterol condition $(P<0.05)$; $\ddagger$ mean value was significantly different from that of the $10 \mathrm{~min}$ reperfusion cholesterol condition $(P<0.001)$. (C) The effect of RPO supplementation on the phosphorylation of $\mathrm{C}-\mathrm{Jun}$ $\mathrm{NH}_{2}$-terminal kinase (JNK) in cholesterol-fed rat hearts subjected to ischaemia and reperfusion. Samples were analysed by Western blotting with phospho-specific antibodies recognising dual phosphorylated MAPK. Data are means for six independent experiments, with their standard errors represented by vertical bars. JNK54: mean value was significantly different from that of the 10 min reperfusion control: ${ }^{\star} P<0.01,{ }^{\star \star} P<0.001$; $†$ mean value was significantly different from that of the $10 \mathrm{~min}$ reperfusion cholesterol condition $(P<0.001)$. JNK46: mean value was significantly different from that of the 10 min reperfusion control: ${ }^{\star} P<0.01$, ${ }^{\star \star} P<0.001$; $†$ mean value was significantly different from that of the $10 \mathrm{~min}$ reperfusion cholesterol condition $(P<0.001)$. 

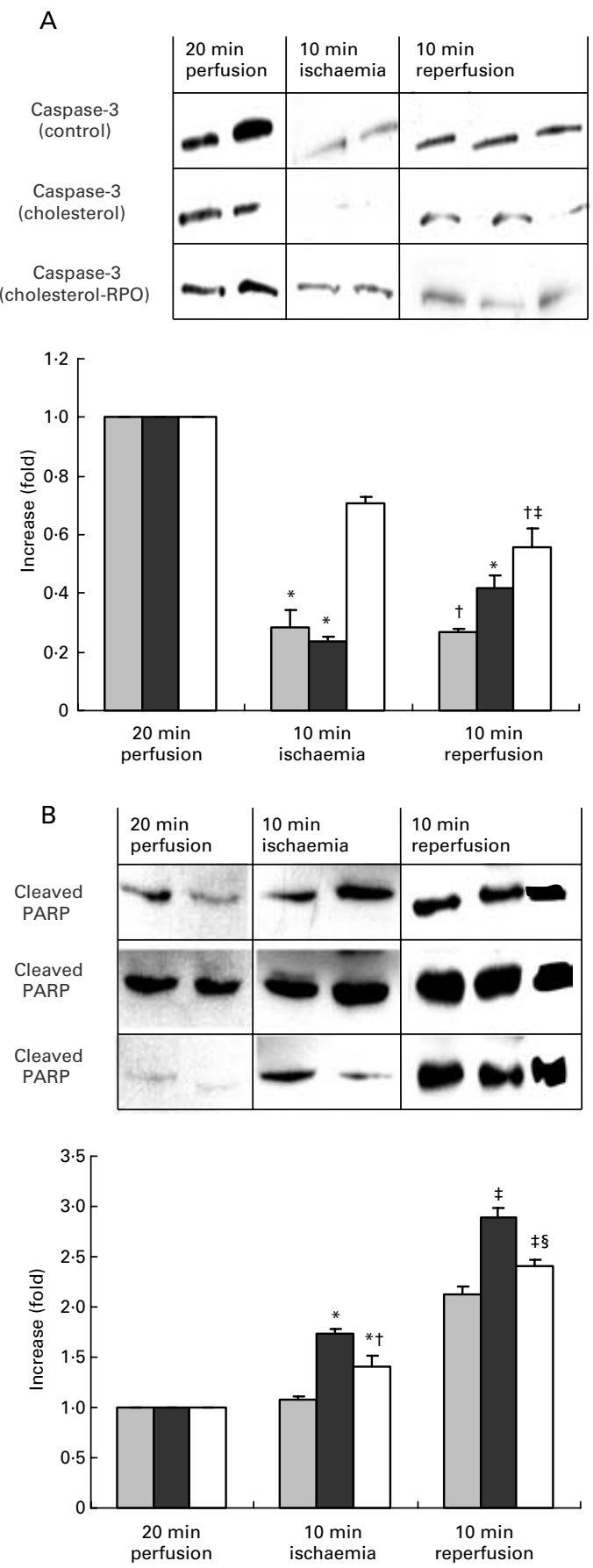

Fig. 4. The effect of red palm oil (RPO) supplementation on caspase-3 activation $(A)$ and poly(ADP-ribose) polymerase (PARP) cleavage (B) in cholesterol-fed rat hearts subjected to ischaemia and reperfusion. Samples were analysed by Western blotting with antibodies recognising cleaved PARP and

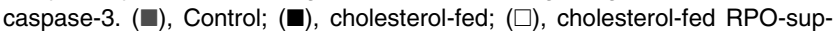
plemented. Data are means for six independent experiments, with their standard errors represented by vertical bars. Caspase-3: ${ }^{*}$ mean value was significantly different from that of the $10 \mathrm{~min}$ ischaemia cholesterol-RPO condition $(P<0.001)$; $†$ mean value was significantly different from that of the $10 \mathrm{~min}$ reperfusion cholesterol condition $(P<0.01)$; $\ddagger$ mean value was significantly different from that of the $10 \mathrm{~min}$ reperfusion control $(P<0.001)$. PARP: * mean value was significantly different from that of the $10 \mathrm{~min}$ ischaemia control $(P<0.001)$; $†$ mean value was significantly different from that of the 10 min ischaemia cholesterol condition $(P<0.001)$; $\ddagger$ mean value was significantly different from that of the 10 min reperfusion control $(P<0.001)$; § mean value was significantly different from that of the $10 \mathrm{~min}$ reperfusion cholesterol condition $(P<0.001)$.
Therefore, RPO might offer an alternative, non-pharmacological strategy to protect the heart against ischaemia-reperfusion-induced injury in the presence of cholesterol, one of the risk factors ultimately leading to IHD.

\section{Acknowledgements}

The present study was supported by the University of Stellenbosch (Subcommittee B), National Research Foundation of South Africa and the Cape Peninsula University of Technology. Carotino Palm Oil was supplied by Carotino SDN BHD (company no. 69046-T), Johore Bahry, Malaysia. We also thank Dirk Bester from the Cape Peninsula University of Technology for his technical support.

\section{References}

Abe J, Baines CP \& Berk BC (2000) Role of mitogen-activated protein kinases in ischaemia and reperfusion injury: the good and the bad. Circ Res 86, 607-609.

Bernabe JC, Tejedo JR, Rincon P, Cahuana GM, Ramirez R, Sobrino F \& Bedoya FJ (2001) Sodium nitroprusside-induced mitochondrial apoptotic events in insulin-secreting RINm5F cells are associated with MAP kinases activation. Exp Cell Res 269, 222-229.

Bhattacharya S, Ray RM \& Johnson LR (2004) Prevention of TNF- $\alpha$ induced apoptosis in polyamine-depleted IEC-6 cells is mediated through the activation of ERK1/2. Am J Physiol 286, G479-G490.

Bilgin-Karabulut A, Ademoglu E, Aydin I, Erer M \& Gokkusu C (2001) Protective effects of vitamins A and E pretreatment in venous ischemia/reperfusion injury. $J$ Reconstr Microsurg 17, 425-429.

Bogoyevitch MA, Gillespie-Brown J, Ketterman AJ, Fuller SJ, BenLevy R, Ashworth A, Marshall CJ \& Sugden PH (1996) Stimulation of the stress-activated mitogen-activated protein kinase subfamilies in perfused heart. p38/RK mitogen-activated protein kinases and c-Jun $\mathrm{N}$-terminal kinases are activated by ischaemia/reperfusion. Circ Res 79, 162-173.

Bonni A, Brunet A, West AE, Datta SR, Takasu MA \& Greenberg ME (1999) Cell survival promoted by the Ras-MAPK signaling pathway by transcription-dependent and -independent mechanisms. Science 286, 1358-1362.

Bradford MM (1976) A rapid and sensitive method for the quantitation of microgram quantities of protein utilizing the principle of protein-dye binding. Anal Biochem 72, 248-254.

Engelbrecht AM, Esterhuyse J, du Toit EF, Lochner A \& van Rooyen J (2006) p38-MAPK and PKB/Akt, possible role players in red palm oil-induced protection of the isolated perfused rat heart? $J$ Nutr Biochem 17, 265-271.

Engelbrecht AM, Niesler C, Page C \& Lochner A (2004) p38 and JNK have distinct regulatory functions on the development of apoptosis during simulated ischaemia and reperfusion in neonatal cardiomyocytes. Basic Res Cardiol 99, 338-350.

Esterhuyse AJ, du Toit EF, Benade AJ \& van Rooyen J (2005) Dietary red palm oil improves reperfusion cardiac function in the isolated perfused rat heart of animals fed a high cholesterol diet. Prostaglandins Leukot Essent Fatty Acids 72, 153-161.

Ferdinandy P (2003) Myocardial ischaemia/reperfusion injury and preconditioning: effects of hypercholesterolaemia/hyperlipidaemia. Br J Pharmacol 183, 283-285.

Ferdinandy P, Szilvassy Z, Horvath LI, Csont T, Csonka C, Nagy E, Szentgyorgyi R, Nagy I, Koltai M \& Dux L (1997) Loss of pacinginduced preconditioning in rat hearts: role of nitric oxide and cholestrol-enriched diet. J Mol Cell Cardiol 29, 3321-3333. 
Golino P, Maroko PR \& Carew TE (1987) The effect of acute hypercholesterolemia on myocardial infarct size and the noreflow phenomenon during coronary occlusion-reperfusion. Circulation 75, 292-298.

Giricz Z, Csonka C, Onody A, Csont T \& Ferdinandy P (2003) Role of cholesterol-enriched diet and the mevalonate pathway in cardiac nitric oxide synthesis. Basic Res Cardiol 98, 304-310.

Hreniuk D, Garay M, Gaarde W, Monia BP, McKay RA \& Cioffi CL (2001) Inhibition of c-Jun N-terminal kinase 1, but not c-Jun Nterminal kinase 2, suppresses apoptosis induced by ischaemia/reoxygenation in rat cardiac myocytes. Mol Pharmacol 59, 867-874.

Kyriakides ZS, Psychari S, Iliodromitis EK, Kolettis TM, Sbarouni E \& Kremastinos DT (2002) Hyperlipidemia prevents the expected reduction of myocardial ischemia on repeated balloon inflations during angioplasty. Chest 121, 1211-1215.

Mackay K \& Mochly-Rosen D (2000) Involvement of a p38 mitogenactivated protein kinase phosphatase in protecting neonatal rat cardiac myocytes from ischaemia. J Mol Cell Cardiol 32, 1585-1588.

Marais E, Genade S, Strijdom H, Moolman JA \& Lochner A (2001) p38 MAPK activation triggers pharmacologically-induced $\beta$-adrenergic preconditioning, but not ischaemic preconditioning. $J \mathrm{Mol}$ Cell Cardiol 33, 2157-2177.

Matsukawa J, Matsuzawa A, Takeda K \& Ichijo H (2004) The ASK1MAP kinase cascades in mammalian stress response. $J$ Biochem (Tokyo) 136, 261-265.

Maulik N, Watanabe M, Zu YL, Huang CK, Cordis GA, Schley JA \& Das DK (1996) Ischemic preconditioning triggers the activation of MAP kinases and MAPKAP kinase 2 in rat hearts. FEBS Lett 396, 233-237.

Meehan AG \& Higgins AJ (1994) Oleate plus oxfenicine improves functional recovery, assessed via an intraventricular balloon, in ischemic-reperfused rat hearts. Ann NY Acad Sci 723, $343-344$.

Minet E, Michel G, Mottet D, Raes M \& Michiels C (2001) Transduction pathways involved in hypoxia-inducible factor- 1 phosphorylation and activation. Free Radic Biol Med 31, 847-855.

Nagendran B, Unnithan UR, Choo YM \& Sundram K (2000) Characteristics of red palm oil, a carotene- and vitamin E-rich refined oil for food uses. Food Nutr Bull 2, 189-194.

Niyongabo A, Youyou A, Leger CL, Descomps B, Ammouche A \& Bellal M (1999) Effects of dietary crude palm oil, fish oil and their association on cholesterol and lipoprotein constants in rats which could be beneficial in humans. Int J Vitam Nutr Res 69 , $330-336$.
Nor Aini \& Suria MSA (2000) Food uses of palm and palm kernel oils. In Adances in Palm Oil Research, pp. 968-1035 [Y Basiron, BS Jalani, KW Chan, editors] Kuala Lumpur: Malaysian Palm Oil Board.

Obata T, Brown GE \& Yaffe MB (2000) MAP kinase pathways activated by stress: the p38 MAPK pathway. Crit Care Med 28, N67-N77.

Park WH, Seol JG, Kim ES, Hyun JM, Jung CW, Lee CC, Binderup L, Koeffler HP, Kim BK \& Lee YY (2000) Induction of apoptosis by vitamin D3 analogue EB1089 in NCI-H929 myeloma cells via activation of caspase 3 and p38 MAP kinase. Br J Haematol 109, 576-583.

Saurin AT, Martin JL, Heads RJ, Foley C, Mockridge JW, Wright MJ, Wang Y \& Marber MS (2000) The role of differential activation of $\mathrm{p} 38$-mitogen-activated protein kinase in preconditioned ventricular myocytes. FASEB J 14, 2237-2246.

Serbinova E, Khavaja S, Catudioc J, Ericson J, Torres Z \& Gapor A (1992) Palm oil vitamin E protects against ischemia/reperfusion injury in the isolated perfused Langendorf heart. Nutr Res 12, S203-S215.

Wang Y, Su B, Sah VP, Brown JH, Han J \& Chien KR (1998) Cardiac hypertrophy induced by mitogen-activated protein kinase kinase 7, a specific activator for c-Jun NH2-terminal kinase in ventricular muscle cells. J Biol Chem 273, 5423-5426.

Weinbrenner C, Liu GS, Cohen MV \& Downey JM (1997) Phosphorylation of tyrosine 182 of p38 mitogen-activated protein kinase correlates with the protection of preconditioning in the rabbit heart. J Mol Cell Cardiol 29, 2383-2391.

Whitmarsh AJ, Yang SH, Su MS, Sharrocks AD \& Davis RJ (1997) Role of p38 and JNK mitogen-activated protein kinases in the activation of ternary complex factors. Mol Cell Biol 17, 2360-2371.

Yue TL, Wang C, Gu JL, Ma XL, Kumar S, Lee JC, Feuerstein GZ, Thomas H, Maleeff B \& Ohlstein EH (2000) Inhibition of extracellular signal-regulated kinase enhances ischaemia/reoxygenation-induced apoptosis in cultured cardiac myocytes and exaggerates reperfusion injury in isolated perfused heart. Circ Res 86, 692-699.

Zhang J, Ping W, Chunrong W, Shou CX \& Keyou G (1997) Nonhypercholesterolemic effects of a palm oil diet in Chinese adults. $J$ Nutr 127, 509S-513S.

Zhang J, Wang C, Dai JH, Chen XS \& Ge K (1997) Palm oil diet may benefit mildly hypercholesterolaemic Chinese adults. Asia Pac J Clin Nutr 6, 22-25. 